\title{
Process and outcome indicators for infection control and prevention in European acute care hospitals in 2011 to 2012 - Results of the PROHIBIT study
}

Sonja Hansen ${ }^{1}$, Frank Schwab ${ }^{1}$, Walter Zingg ${ }^{2}$, Petra Gastmeier ${ }^{1}$, the PROHIBIT study group ${ }^{3}$

1. Charité - Universitätsmedizin Berlin, corporate member of Freie Universität Berlin, Humboldt-Universität zu Berlin, and Berlin Institute of Health, Institute for Hygiene and Environmental Medicine, Berlin, Germany

2. University of Geneva Hospitals, Infection Control Programme, Switzerland

3. The members of the PROHIBIT study group are listed at the end of the article

Correspondence: Sonja Hansen (sonja.hansen@charite.de)

Hansen Sonja, Schwab Frank, Zingg Walter, Gastmeier Petra, the PROHIBIT study group. Process and outcome indicators for infection control and prevention in European acute care hospitals in 2011 to 2012 - Results of the PROHIBIT study. Euro Surveill. 2018;23(21):pii=1700513. https://doi.org/10.2807/156o-7917. ES.2018.23.21.1700513

Introduction: Hospitals from 24 European countries were asked for information on infection prevention and control (IPC) indicators as part of the Prevention of Hospital Infections by Intervention and Training (PROHIBIT) survey. Methods: Leading IPC personnel of 297 hospitals with established healthcare-associated infection ( $\mathrm{HCAl}$ ) surveillance provided information on local surveillance and feedback by using a questionnaire. Results: Most hospitals focused on bloodstream infection (BSI) $(n=251)$ and surgical site infection (SSI) $(n=254)$, with a SSI post-discharge surveillance in 148 hospitals. As part of the HCAl surveillance, meticillin-resistant Staphylococcus aureus (MRSA) was the leading multidrug-resistant organism (MDRO) under surveillance. Seventy-nine per cent of hospitals $(n=236)$ monitored alcohol-based hand rub (ABHR) consumption. Feedback to the local IPC committees mainly included outcome data on HCAI $(n=259 ; 87 \%)$ and MDRO among HCAI $(n=245 ; 83 \%)$; whereupon a feedback of MDRO data depended on hospital size $(p=0.012)$. Discussion/conclusion: Objectives and methods of surveillance vary across Europe, with BSI, SSI and MRSA receiving considerably more attention than indicators such as pneumonia and urinary tract infection, which may be equally important. In order to maximise prevention and control of HCAI and MDRO in Europe, surveillance should be further improved by targeting relevant HCAI. The role of feedback should be explored in more detail.

\section{Introduction}

Based on the results of the first European point prevalence survey (PPS) in 2011-12 an estimated 3.2 million patients acquire a healthcare-associated infection (HCAl) in acute care hospitals in Europe every year [1]. The most common types of HCAI are surgical site infections (SSI), urinary tract infections (UTI), pneumonia
(PN), bloodstream infections (BSI), and gastrointestinal infections, with Clostridium difficile infection (CDI) accounting for a high proportion in the latter. HCAls result in increased morbidity and mortality, and emerging antibiotic resistance complicates their treatment. The cumulative burden of HCAls is higher than the total burden of other communicable diseases in Europe [2].

Surveillance as the "ongoing systematic collection and analysis of health data for the planning, implementation, and evaluation, of public health practice' [3] is a key measure in HCAl prevention and control. Even in the absence of specific prevention actions, surveillance and feedback of outcome indicators decrease HCAl by raising awareness for the issue among healthcare professionals [4-7]. Surveillance, preferably as part of a network, was identified as one of the key components in effective HCAl prevention and an important tool for monitoring the effectiveness of prevention and control measures by the 'Systematic Review and Evidencebased Guidance on Organization of Hospital Infection Control Programmes' (SIGHT) project [8].

Since the 1990s, many European countries have been developing national surveillance networks, either by applying the United States' (US) Centers for Disease Control and Prevention (CDC) National Nosocomial Infection Surveillance/National Healthcare Safety Network (NNIS/NHSN) protocol, or by using adapted methods to better take into account local diagnostic practices [9]. In 2010, the European Centre for Disease Prevention and Control (ECDC) established the Healthcare-Associated Infections surveillance Network (HAl-Net), integrating the Hospitals in Europe Link for Infection Control through Surveillance (HELICS) project 
(2000-4) and the Improving Patient Safety in Europe (IPSE) network (2005-8) [10].

Surveillance of alcohol-based hand rub (ABHR) consumption has become a mandatory quality indicator with public reporting in France since 2006 [11], and was integrated into the national Krankenhaus-InfektionsSurveillance-System (KISS) in 2008 in Germany [12]. Furthermore, national strategies on measuring hand hygiene compliance by direct observation have been organised in a number of European countries [13].

Aspects of specific surveillance activities in European hospitals were obtained as part of the Prevention of Hospital Infections by Intervention and Training (PROHIBIT) project. The PROHIBIT survey was conducted as the first pan-European survey on infection prevention and control (IPC) in order to describe which IPC recommendations are actually being used across Europe and to provide information on gaps in hospitals' IPC policies and practices for policymakers, hospital managers and healthcare workers for further improvement of HCAl prevention. This article summarises data on findings from 24 European countries.

\section{Methods}

Participating countries and hospitals

ECDC national contact points (NCPS) and IPC experts of European countries outside of the European Union (EU) were invited to organise national polls. The NCPS invited national hospitals for participation between September 2011 and March 2012. Participation in the PROHIBIT survey was based mainly on hospital interest rather than on a systematic sampling process.

\section{Survey description}

The survey was developed by an interdisciplinary group and discussed with the HAI-Net representatives. It included four questionnaires in order to assess IPC structure and process indicators (i) at the hospital level, (ii) in intensive care units (ICU), (iii) in nonICU medical wards and (iv) in non-ICU surgical wards. Questionnaires addressed organisation and activities of IPC at those various levels.

The complete method of the survey and the characteristics of the participating hospitals are described in more detail elsewhere [14].

For the present analysis, data on HCAI surveillance, process and outcome indicators (e.g. ABHR consumption, $\mathrm{HCAl}$ ), direct hand hygiene observations, feedback practices, and persons performing surveillance are described at hospital level. Hospitals were asked whether the following multidrug-resistant organisms (MDRO) were monitored among HCAI: meticillin-resistant Staphylococcus aureus (MRSA), vancomycin-resistant Enterococci (VRE), extendedspectrum beta-lactamase (ESBL)-producing Enterobacteriaceae, carbapenem nonsusceptible or carbapenemase-producing Enterobacteriaceae, carbapenem-resistant Pseudomonas aeruginosa and multiresistant Acinetobacter baumannii.

Local IPC professionals were also asked to provide data on hospital characteristics such as status (public/private) and size of the hospital (number of beds), and the full time equivalent (FTE) of infection control personnel.

Furthermore, country characteristics such as the United Nations (UN) European geographical region, and healthcare expenditure (HCE) as share on the national gross domestic product (GDP) were collected $[15,16]$.

\section{Data analysis}

Descriptive data analysis was performed and results summarised as totals and strata of the following four parameters: hospital size (small: $\leq 300$ beds; medium: 301-600 beds; large:>600 beds), UN European geographical regions, full-time-equivalent (FTE) infection control nurses (ICN) (internal staff and external staff) per 1,000 acute care hospital beds $\leq />$ the median of all participating hospitals (3.72 FTE ICN/1,000 beds) and HCE as share on GDP $[15,16]$. HCE was modelled as a dichotomous variable and considered low or high if below or above the European mean HCE of $9 \%$. Differences in the process and outcome indicators between the strata of the four parameters described above were tested by logistic regressions models. In the regression analysis with indicator parameters as outcome, only the independent variables were included in the generalised estimating equation (GEE) models, adjusting for cluster effects by country. A two-sided $\mathrm{p}$ value 0.05 in the type III test was considered significant. All analyses were performed with SPSS (IBM SPSS statistics, Somer, NY, US) and SAS (SAS Institute, Cary, NC, US).

\section{Results}

\section{Participating countries and hospitals}

Of 32 invited countries, 24 participated (Table 1) with 309 acute care hospitals. From all 309 acute care hospitals participating in the PROHIBIT survey, 297 hospitals (96\%) had some method of HCAl surveillance in place. Hospitals with HCAl surveillance had a median of 426 beds (interquartile range (IQR): 260-277), and were most often public hospitals ( 253 hospitals, $85 \%$ ).

\section{Medical conditions/disease outcome where HCAI surveillance applies}

Surveillance in the hospitals mainly focused on SSI and BSI, and less often on PN, CDI and UTI (Table 2). Surveillance of UTI depended on countries' HCE with significantly higher proportions in countries with low HCE. For PN, significant differences were observed in accordance to the hospital size, with more medium to large hospitals having PN surveillance in place compared to smaller hospitals. Significantly more hospitals from countries with low HCE performed hospital-wide 
surveillance of PN and UTI. Hospital-wide surveillance of BSI varied significantly with the UN regions.

Surveillance of CDI was reported most often by hospitals in Northern Europe and more often by hospitals in countries with high HCE; but these differences were not statistically significant.

Hip prosthesis implantation (HPRO) was the most common indicator operation of SSI surveillance with higher percentages in countries with high HCE; but these differences were not statistically significant. In 148 of 254 hospitals with SSI surveillance $(58 \%)$, post-discharge surveillance (PDS) was in place.

\section{Multidrug-resistant organisms surveyed among HCAIs}

MRSA was the most commonly observed MDRO among HCAI in almost all hospitals $(n=273)$, followed by ESBL-producing Enterobacteriaceae $(n=243)$ and VRE $(\mathrm{n}=228)$. Multidrug-resistant Acinetobacter baumannii surveillance was reported by 204 hospitals, carbapenem nonsusceptible or carbapenemase-producing Enterobacteriaceae by 189 hospitals, and carbapenemresistant Pseudomonas aeruginosa by 185 hospitals.

\section{Monitoring hand hygiene compliance}

Consumption of $A B H R$ and hand hygiene compliance was observed in $79 \%$ and $78 \%$ of the hospitals, respectively (Table 2). Hospitals in countries in Northern Europe preferred monitoring hand hygiene compliance to monitoring ABHR consumption, while hospitals in countries in Western Europe preferred monitoring ABHR consumption to monitoring hand hygiene compliance (Table 2).

\section{Operators involved in HCAI surveillance}

Table 3 summarises the operators involved in HCAI surveillance. Most surveillance activities are performed by IPC personnel. Although the overall numbers are low, it appeared that hospitals in countries of Northern Europe had a higher percentage of specific staff dedicated to surveillance of HCAl compared to other regions. In almost half of the hospitals, data on HCAI were collected by IPC personnel, whereas surveillance exclusively performed by ward personnel was by reported by $9 \%$ of all hospitals, and $18 \%$ of hospitals in Eastern Europe.

\section{Feedback on the HCAI situation and hand} hygiene compliance

In almost all hospitals, healthcare workers (HCW) received feedback on HCAI $(n=106$ more thantwice a year, $n=61$ twice a year, $n=115$ once a year, $n=15$ less than once a year). Of the 236 hospitals that performed ABHR consumption surveillance, 200 hospitals provided feedback at least once a year $(n=41$ more thantwice a year, $n=32$ twice a year, $n=127$ once a year), while in 35 hospitals feedback was given less than once a year and for one hospital information on the frequency of feedback was unavailable. Concerning direct hand hygiene compliance observations, 152 hospitals provided immediate feedback to the observed personnel and 131 hospitals provided a later summary feedback.

As shown in the Figure, IPC committees mainly received data on $\operatorname{HCAI}(n=259 ; 87 \%)$ and the proportion of MDRO among HCAls $(n=245 ; 83 \%)$ but less often on hand hygiene performance indicators. Feedback on MDRO among HCAls was most often provided in larger hospitals $(p=0.012)$. IPC committees in hospitals with ICN rates above the European median received significantly more often feedback on hand hygiene compliance data compared to IPC committees in hospitals with ICN rates below the median $(p=0.039)$. Feedback on hand hygiene performance (ABHR consumption and/ or hand hygiene compliance) was significantly more often provided in countries with high HCE $(p=0.042)$. No feedback was given to IPC committees in $23(8 \%)$ hospitals.

\section{Discussion}

To our knowledge, the data of the PROHIBIT survey offer the first broad analysis of $\mathrm{HCAl}$ and MDRO surveillance activities in European acute care hospitals. The findings show that content and methods of surveillance and the role of feedback vary widely across Europe. Hospitals focused more frequently on the surveillance of outcome indicators as BSI and SSI than on PN, CDI or UTI. This may be due to numerous success stories of BSI and SSI preventability, which raised hospitals' awareness towards these two infection types [17-19].

Fifty-eight per cent of hospitals with SSI surveillance reported to have PDS in place. Such additional surveillance as described by Woelber et al. in 2016, partly prevents under-reporting of SSI in Europe [20]. The finding that HPRO is the most common indicator procedure for SSI surveillance corresponds to the results of ECDC's HAI-Net surveillance of SSI with HPRO being the most frequently reported type of surgery, representing $33 \%$ of all operations in 2010-11 [21].

Nevertheless, successful preventability of HCAls such as PN and UTI has also been described [22,23] and data of the PPS from 2011-12 indicate that respiratory tract infections and UTI are common HCAls all over Europe. Interestingly, UTI surveillance was significantly more frequently reported in countries with low HCE. Data of the PPS for countries with high HCE however, showed frequencies of UTIs up to $31 \%$, indicating that patients hospitalised in such countries are also at risk for this type of HCAI [1].

Resources are limited; and thus, priorities must be made in HCAl surveillance, even if a broad surveillance strategy including process and outcome indicators is considered helpful to tailor intervention activities for HCAI prevention. Prospective hospital-wide HCAl surveillance is resource-intensive, and in this sense, it was surprising to find that the proportion of hospitals 


\section{FIGURE}

Feedback of surveillance data to the infection control committees in European acute care hospitals with established healthcare-associated infection surveillance, stratified by healthcare expenditure, infection control nurse rate, United Nation regions and hospital size - The Prevention of Hospital Infection by Intervention and Training (PROHIBIT) survey, 2011-2012 ( $\mathrm{n}=297$ hospitals $)$

\section{A. Healthcare expenditure ${ }^{a}$}

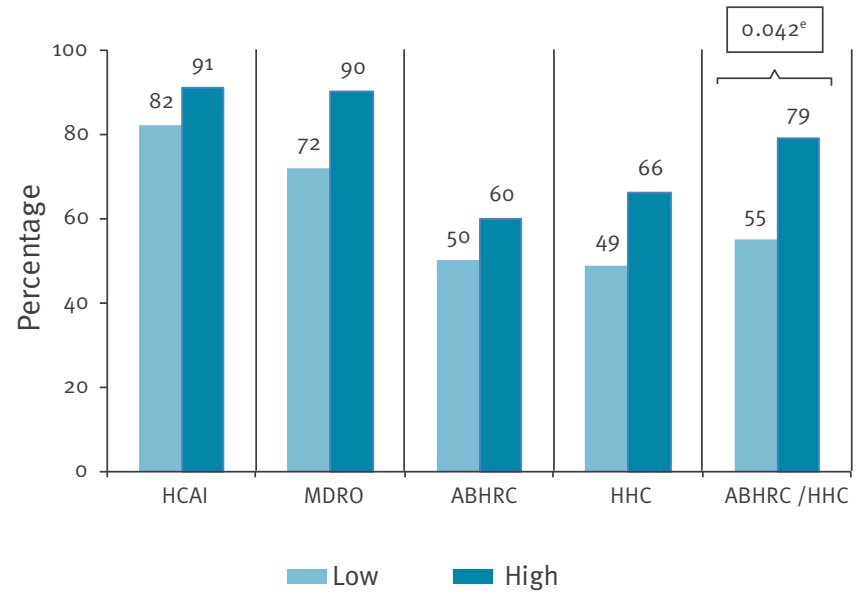

\section{United Nations regions ${ }^{c}$}

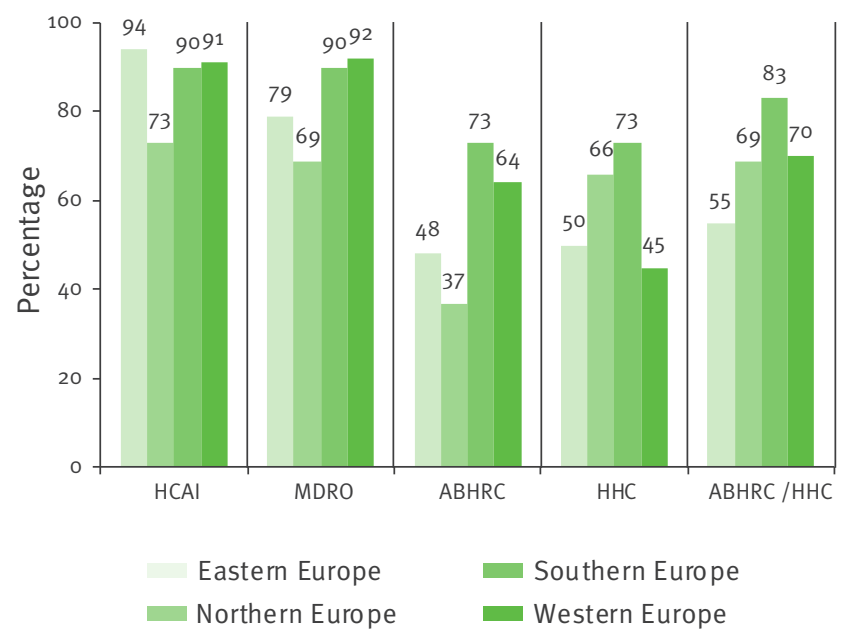

B. Infection control nurse rate

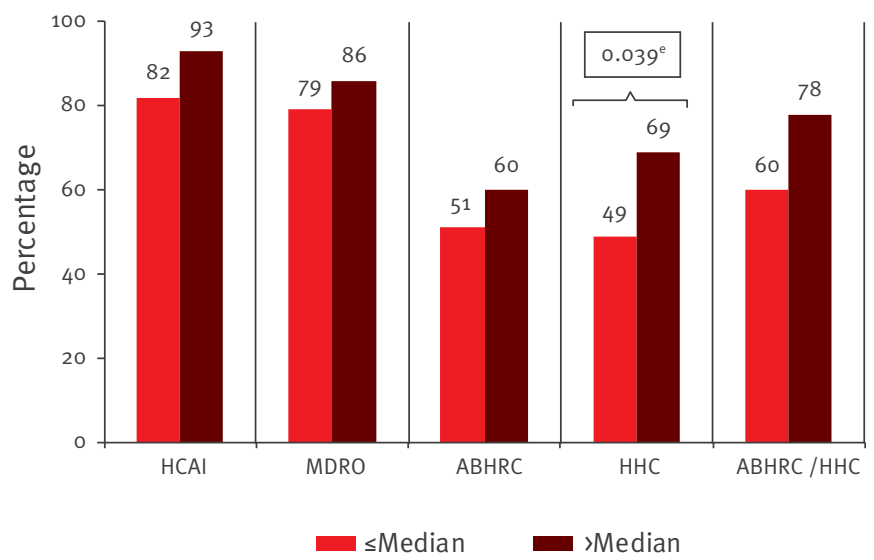

D. Hospital size

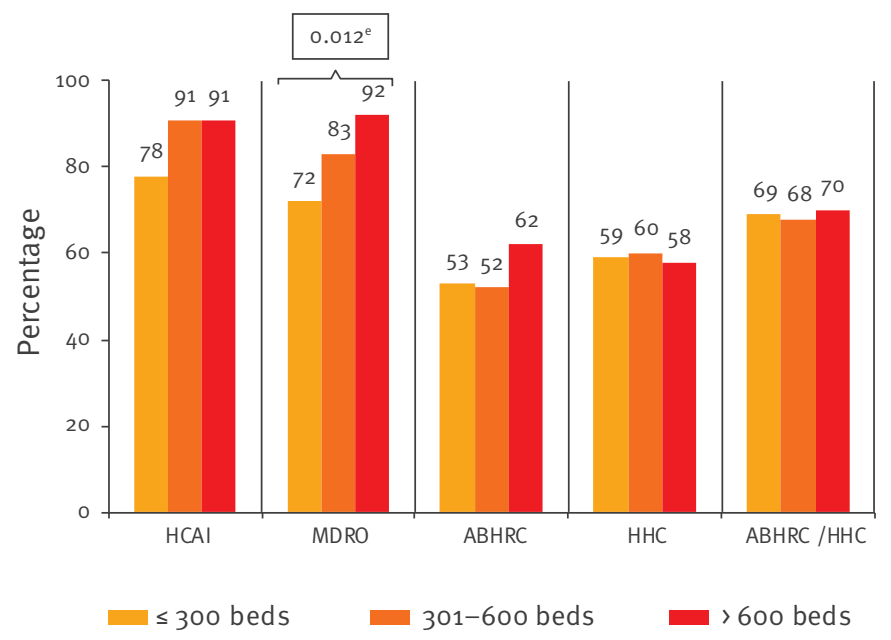

ABHRC: alcohol-based hand rub consumption; ABHRC/HHC: ABHRC and/or hand hygiene compliance; FTE: full time equivalent; HCAI: healthcare-associated infections; HCE: healthcare expenditure; HHC: hand hygiene compliance; ICN: infection control nurse; MDRO: multidrug-resistant organisms.

${ }^{a}$ Low/high HCE defined as the share of the gross domestic product $\leq /$ the European mean in 2010 (9\%) [16]; low HCE ( $\left.\mathrm{n}=127\right)$, high HCE $(n=170)$.

${ }^{b}$ FTE ICN (internal staff and external staff) per 1,000 acute care hospital beds $\leq />$ the median of all participating hospitals (3.72 FTE ICN/1,000 beds); ‘ $\leq$ median' $(n=150)$, ‘> median' $(n=147)$.

${ }^{c}$ Geographical regions according to United Nations grouping [15]; Eastern Europe $(n=82)$, Northern Europe $(n=70)$, Southern Europe ( $\left.=81\right)$, Western Europe $(n=64)$.

' Hospital size according to number of acute care beds; ' $\leq 300$ beds' $(n=87)$, ‘301-600 beds' ( $n=109)$, '> 600 beds' ( $n=98)$; information available for 294 hospitals.

e $\mathrm{P}$ values were calculated by logistic regression using generalised estimating equations, which account for cluster effects by country. 
Distribution of hospitals providing data on healthcare-associated infection surveillance and national healthcare expenditure as part of the gross domestic product by country - the Prevention of Hospital Infection by Intervention and Training (PROHIBIT) survey, Europe, 2011-2012 ( $\mathrm{n}=297$ hospitals)

\begin{tabular}{|c|c|c|c|c|}
\hline \multirow{2}{*}{ United Nations region ${ }^{\mathrm{a}}$} & \multirow{2}{*}{ Country } & \multirow{2}{*}{ Total HCE as $\%$ of GDPb } & \multicolumn{2}{|c|}{ Number of participating hospitals } \\
\hline & & & $n$ & $\%$ \\
\hline \multirow{8}{*}{ Northern Europe, $n=70$} & Finland & 8.9 & 11 & 3.7 \\
\hline & Ireland & 9.2 & 12 & 4 \\
\hline & Latvia & 6.8 & 7 & 2.4 \\
\hline & Lithuania & 7 & 13 & 4.4 \\
\hline & Sweden & 9.6 & 6 & 2 \\
\hline & United Kingdom, England & \multirow{3}{*}{9.6} & 5 & 1.7 \\
\hline & United Kingdom, Scotland & & 3 & 1 \\
\hline & United Kingdom, Wales & & 13 & 4.3 \\
\hline \multirow{4}{*}{ Eastern Europe, $n=82$} & Bulgaria & 7.2 & 19 & 6.4 \\
\hline & Hungary & 7.8 & 30 & 10.1 \\
\hline & Poland & 7 & 9 & 3 \\
\hline & Slovakia & 9 & 24 & 8.1 \\
\hline \multirow{6}{*}{ Southern Europe, $n=81$} & Croatia & 7.8 & 5 & 1.7 \\
\hline & Italy & 9.3 & 18 & 6.1 \\
\hline & Malta & 8.6 & 1 & 0.3 \\
\hline & Portugal & 10.7 & 26 & 8.8 \\
\hline & Slovenia & 9 & 8 & 2.7 \\
\hline & Spain & 9.6 & 23 & 7.7 \\
\hline \multirow{6}{*}{ Western Europe, $n=64$} & Austria & 11 & 8 & 2.7 \\
\hline & Belgium & 10.5 & 5 & 1.7 \\
\hline & France & 11.6 & 8 & 2.7 \\
\hline & Germany & 11.6 & 29 & 9.8 \\
\hline & Switzerland & 11.4 & 6 & 2 \\
\hline & The Netherlands & 12 & 8 & 2.7 \\
\hline All & NA & NA & 297 & 100 \\
\hline
\end{tabular}

GDP: gross domestic product; HCE: healthcare expenditure; NA: not applicable.

${ }^{a}$ Geographical regions according to United Nations grouping [15].

${ }^{\mathrm{b}} \mathrm{HCE}$ as the share of the GDP [16].

with hospital-wide PN and UTI surveillance was significantly higher in hospitals from countries with low HCE compared to hospitals from countries with high HCE. Generally, hospital-wide surveillance of HCAI is time-consuming and repeated PPSs on HCAI or an automated surveillance linking administrative data and clinical databases including microbiology may be a better approach.

Large healthcare-associated outbreaks of CDI in the first decade of 2000 sparked increased awareness of CDI prevention in Europe, and resulted in European guidelines on CDI prevention in 2008, recommending CDI surveillance [24]. Data of our survey showed that fewer hospitals in countries with low HCE established CDI surveillance, indicating that surveillance activities as a whole may be influenced by financial constraints in Europe. The low level of CDI surveillance activities in these hospitals may be due to absent national CDI surveillance systems, but also to a lack of diagnostic testing and missing awareness as a consequence $[1,25]$. The high number of hospitals performing CDI surveillance in Northern Europe can be seen as a consequence of public reporting on CDI in the United Kingdom (UK). The new European protocol of CDI surveillance for acute care hospitals, which was developed in 2013, offers a standardised cross-country surveillance, with the option of integrating clinical and molecular data, and can contribute to enhanced monitoring of CDI in all parts of Europe [26].

Although low, still $10 \%$ of hospitals in Europe, and nearly $20 \%$ of hospitals in Eastern Europe, collect HCAI data by ward personnel only. This method of case finding can be interpreted as a more passive rather than active surveillance, with potential bias of under-reporting. As recommended by the Association for Professionals in Infection Control and Epidemiology (APIC), appropriate education to apply infection surveillance definitions or 
TABLE 2

Surveillance of process and outcome indicators in European acute care hospitals, stratified by healthcare expenditure, United Nation regions, hospital size, rate of infection control nurses - the Prevention of Hospital Infection by Intervention and Training (PROHIBIT) survey, 2011-2012 ( $\mathrm{n}=297$ hospitals)

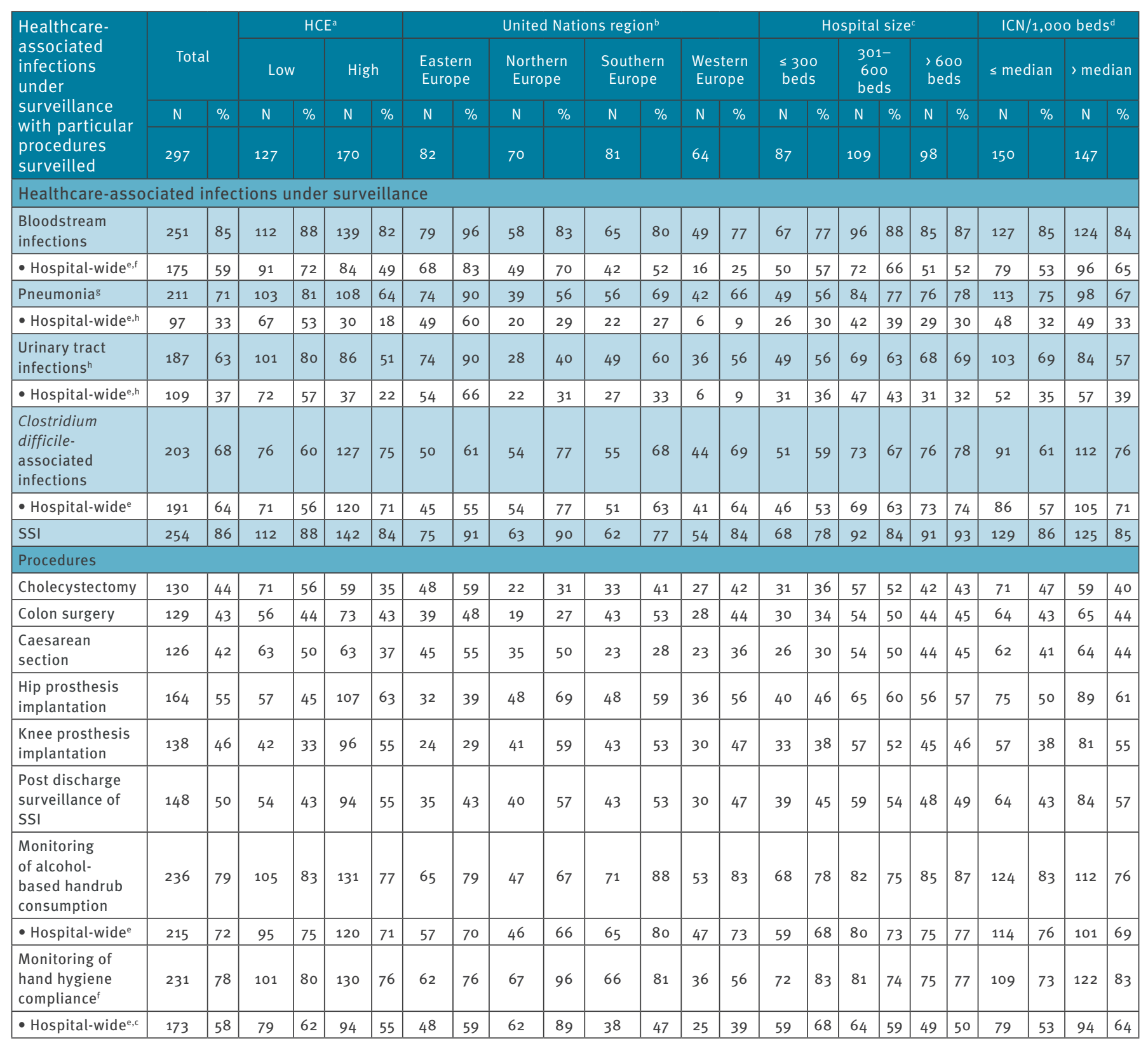

FTE: full time equivalent; HCE: healthcare expenditure; ICN: infection control nurse; SSI: surgical site infection.

$P$ values were calculated by logistic regression using generalised estimating equations, which account for cluster effects by country.

a Low/high HCE defined as the share of the gross domestic product $\leq />$ the European mean in 2010 (9\%) [16].

${ }^{b}$ Geographical regions according to United Nations grouping [15]; Eastern Europe, Northern Europe, Southern Europe, Western Europe.

${ }^{c}$ Hospital size according to number of acute care beds; $\leq 300$ beds, $301-600$ beds, $>600$ beds; information available for 294 hospitals.

d FTE ICN (internal staff and external staff) per 1,000 acute care hospital beds $\leq />$ the median of all participating hospitals ( 3.72 FTE ICN/1,000 beds).

e Hospital-wide describes that the surveillance takes place in all units and wards of the hospital.

${ }^{f}$ Differences between United Nations regions $\mathrm{p}<0.05$.

${ }^{g}$ Differences between $\leqslant 300$ beds / $301-600$ beds / $>600$ beds; $p<0.05$.

${ }^{\mathrm{h}}$ Differences between low/high HCE; $\mathrm{p}<0.05$.

to perform detailed risk factor collection is indispensable [27].

In addition to the surveillance of outcomes, many hospitals assess data on hand hygiene performance indicators. Monitoring process indicators and assessment of adherence to IPC measures such as hand hygiene observation, enables hospitals to identify gaps and improve adherence to IPC measures more promptly than by focusing on outcome data alone. Interestingly, our study identified a discrepancy between a relatively high number of hospitals monitoring ABHR consumption and a relatively low number of hospitals giving feedback on ABHR consumption data to their IPC 


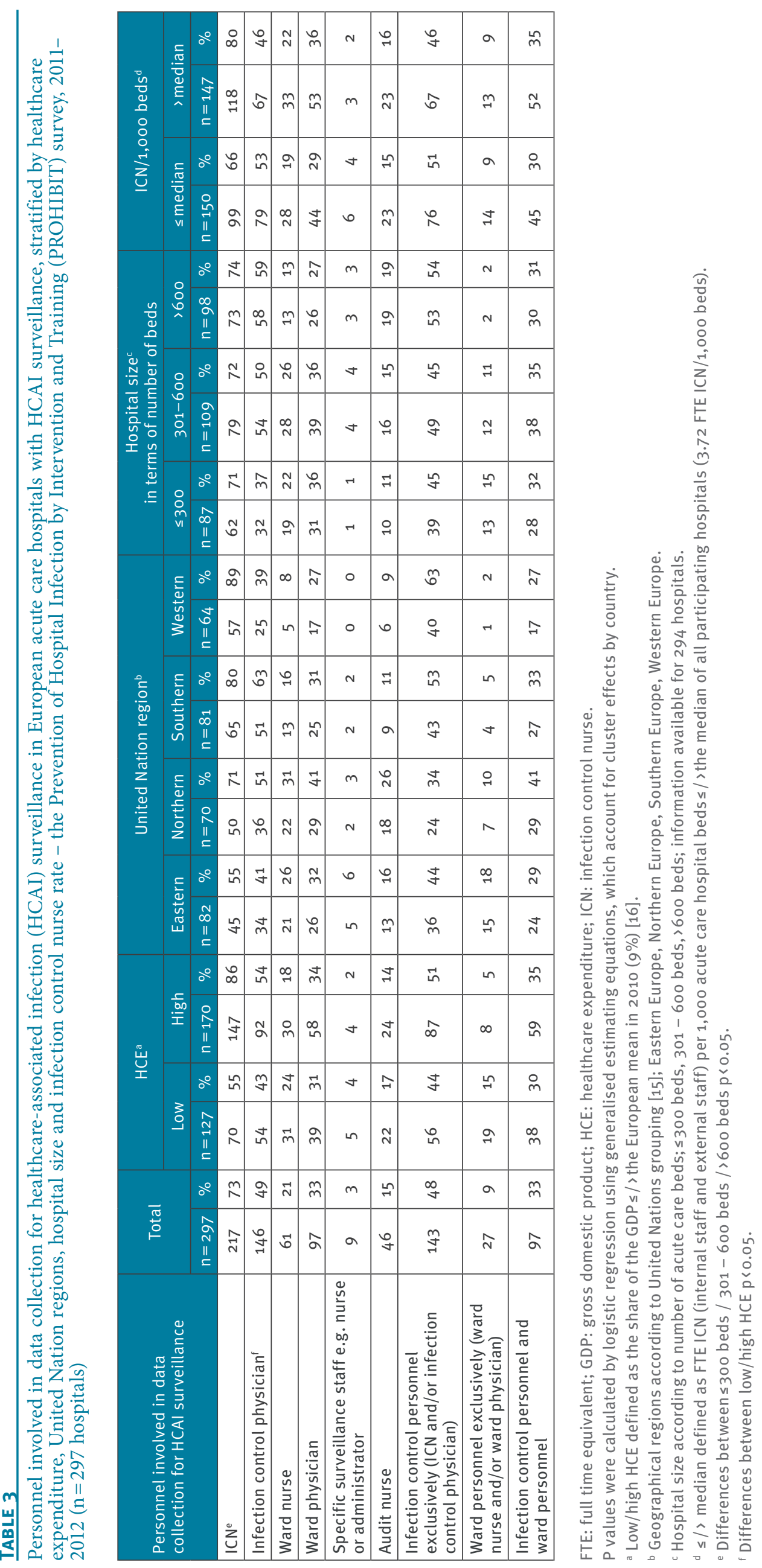


committees. This may be due the fact that many hospitals start surveillance activities focusing on outcome indicators and still work on the feedback of these indicators rather on reporting ABHR. In addition, ABHR data are often collected yearly, and thus, may be reported less frequently to IPC committees. On the other hand, process indicators are better candidates to be used for a realistic target-setting both at ward and hospital level. Reference data on these indicators facilitate inter-hospital comparison to support improving their processes [28]. Europe-wide surveys as the ECDC-PPS or PROHIBIT $[1,14]$ already offer reference data on factors such as IPC personnel or isolation capacities and future projects may generate more, possibly stratified reference data for relevant structural and process IPC parameters.

In order to alter their behaviour in HCAl prevention HCWs have to be aware of the problem of HCAl in their setting. Data of our survey indicate that HCWs do receive feedback on HCAI rates in order to raise awareness. However, more research is needed to explore how surveillance data are communicated and perceived, and how this process can be further optimised. Feedback of data may be combined with behaviourally informed approaches such as the setting of long-term goals and encouraging involvement/participation of HCWs for creating local ownership and reflection on achievements and further activities.

Since successful implementation of IPC measures requires the participation of $\mathrm{HCWs}$ and other stakeholders, feedback to members of the IPC committee is essential. Especially in smaller hospitals, feedback is not always established yet. In which way the size of a hospital influences feedback of MDRO data to hospitals' stakeholders cannot be fully answered. It can be speculated that larger hospitals see more MDROs, and thus, data are perceived more relevant, particularly because they care more frequently for patients with severe and/or chronic diseases.

In the future, all hospitals' IPC committees should be encouraged to work with MDRO data in order to address supporting organisational factors such as leadership support and communication in MDRO transmission prevention and antibiotic stewardship programmes $[29,30]$.

The current survey gives insight into established surveillance activities of European hospitals. However, there are some limitations:

Participation in the survey was voluntary, and thus, based mainly on hospitals' interest rather than on a randomised sampling process. Therefore, the data may have overestimated surveillance activities in European hospitals. A randomly selected sample would have improved representation of hospitals in Europe. However, the questionnaire could not have been imposed on hospitals, and thus, data quality and the number of participating hospitals might have been lower.

The UN geographical regions are not homogeneous in terms of GDP, healthcare organisation and culture. However, by also reporting data in reference to countries' HCE, we tried to take into account such heterogeneity.

Our findings show that objectives and methods of surveillance vary across Europe. Some outcome indicators, such as BSI, SSI and MRSA, seem to receive considerably more attention than others that are equally important, such as PN, UTI or CDI.

Hospitals' IPC committees mainly receive data on outcome indicators as HCAI and MDRO, but less often on process indicators as hand hygiene performance indicators.

In order to better address prevention of HCAI and antimicrobial resistance in Europe surveillance should be further improved by targeting all relevant $\mathrm{HCAl}$ and MDRO and providing active surveillance by trained personnel. To what extent surveillance of process indicators prevent HCAI must be further analysed. In addition, the role of feedback and behaviourally informed approaches should be explored in more detail.

\section{The PROHIBIT study group}

Pittet Didier, Zingg Walter, Sax Hugo, Gastmeier Petra, Hansen Sonja, Grundmann Hajo, van Benthem Birgit, van der Kooi Tjallie, Dettenkofer Markus, Martin Maria, Richet Hervé, Szilágyi Emese, Heczko Piotr, Holmes Alison, Kyratsis Yannis, Ahmad Raheelah, Allegranzi Benedetta, Magiorakos Anna-Pelagia, Cookson Barry, Wu Albert.

\section{Acknowledgements}

The authors would like to thank all participating hospitals for their invaluable input and the following colleagues for their support and organization of the survey: Angel Asensio, Pascal Astagneau, Birgit Van Benthem, Ermira Tartari Bonnici, Ana Budimir, Karen Burns, Barry Cookson, Ana Cristina Costa, Elina Dimina, Uga Dumpis, Greta Gailiene, Michiyo Iwami, Irena Klavs, Tommi Kärki, Andrea Kološova, Andrea Kurcz, David Nicholas Looker, Outi Lyytikäinen, Maria Luisa Moro, Karl Merten, Enrico Ricchizzi, Lisa Ritchie, Kestutis Rudaitis, Emese Szilágyi, Jadwiga Wójkowska, Tjallie van der Kooi, Rossitza Vatcheva-Dobrevska, Inga Zetterqvist.

Funding sources:

PROHIBIT was funded by the European Union's Seventh Framework Programme (FP7), Grant No. 241928.

\section{Conflict of interest}

None declared.

Authors' contributions

Didier Pittet, Walter Zingg, Hugo Sax, Petra Gastmeier, Sonja Hansen, Hajo Grundmann, Birgit van Benthem, Tjallie 
van der Kooi, Markus Dettenkofer, Maria Martin, Hervé Richet, Emese Szilágyi, Piotr Heczko, Alison Holmes, Yannis Kyratsis, Raheelah Ahmad, Benedetta Allegranzi, AnnaPelagia Magiorakos, Barry Cookson and Albert Wu contributed to the design of the PROHIBIT study.

Petra Gastmeier led the survey (Work package 3 of PROHIBIT).

Sonja Hansen managed and coordinated the survey, Frank Schwab analysed the data. Petra Gastmeier, Sonja Hansen, Frank Schwab and Walter Zingg interpreted the results. Sonja Hansen wrote the manuscript. Petra Gastmeier, Frank Schwab and Walter Zingg reviewed and commented on the manuscript.

\section{References}

1. European Centre for Disease Prevention and Control (ECDC). Point prevalence survey of healthcare-associated infections and antimicrobial use in European acute care hospitals. 2011-2012. Stockholm: ECDC; 2013. [Accessed 24Jul 2017]. Available from: http://www.ecdc.europa.eu/en/publications/ Publications/healthcare-associated-infections-antimicrobialuse-PPS.pdf

2. Cassini A, Plachouras D, Eckmanns T, Abu Sin M, Blank HP, Ducomble T, et al. Burden of Six Healthcare-Associated Infections on European Population Health: Estimating Incidence-Based Disability-Adjusted Life Years through a Population Prevalence-Based Modelling Study. PLoS Med. 2016;13(10):e1002150. https://doi.org/10.1371/journal. pmed.1002150 PMID: 27755545

3. Langmuir AD. The surveillance of communicable diseases of national importance. N Engl J Med. 1963;268(4):182 92. https://doi.org/10.1056/NEJM196301242680405 PMID: 13928666

4. Haley RW, Culver DH, White JW, Morgan WM, Emori TG, Munn VP, et al. The efficacy of infection surveillance and control programs in preventing nosocomial infections in US hospitals. Am J Epidemiol. 1985;121(2):182-205. https://doi.org/10.1093/ oxfordjournals.aje.a113990 PMID: 4014115

5. Gastmeier P, Schwab F, Sohr D, Behnke M, Geffers C. Reproducibility of the surveillance effect to decrease nosocomial infection rates. Infect Control Hosp Epidemiol. 2009;30(10):993-9. https://doi.org/10.1086/605720 PMID: 19719414

6. Gaynes R, Richards C, Edwards J, Emori TG, Horan T, Alonso Echanove J, et al. Feeding back surveillance data to prevent hospital-acquired infections. Emerg Infect Dis. 2001;7(2):295-8. https://doi.org/10.3201/eid0702.010230 PMID: 11294727

7. Gastmeier P, Sohr D, Schwab F, Behnke M, Zuschneid I, Brandt C, et al. Ten years of KISS: the most important requirements for success. J Hosp Infect. 2008;70(Suppl 1):11-6. https://doi. org/10.1016/S0195-6701(08)60005-5 PMID: 18994676

8. Zingg W, Holmes A, Dettenkofer M, Goetting T, Secci F, Clack $L$, et al. systematic review and evidence-based guidance on organization of hospital infection control programmes (SIGHT) study group. Hospital organisation, management, and structure for prevention of health-care-associated infection: a systematic review and expert consensus. Lancet Infect Dis. 2015;15(2):212-24. https://doi.org/10.1016/S14733099(14)70854-0 PMID: 25467650

9. Gastmeier P. European perspective on surveillance. J Hosp Infect. 2007;65(Suppl 2):159-64. https://doi.org/10.1016/ S0195-6701(07)60036-X PMID: 17540263

10. European Centre for Disease Prevention and Control (ECDC). Healthcare-associated Infections Surveillance Network (HAI-Net). Stockholm: ECDC. [Accessed 24 Jul 2017]. Available from: https://ecdc.europa.eu/en/about-us/ partnerships-and-networks/disease-and-laboratory-networks/ hai-net

11. Carlet J, Astagneau P, Brun-Buisson C, Coignard B, Salomon $V$, Tran B, et al. French National Program for Prevention of Healthcare-Associated Infections and Antimicrobial Resistance. French national program for prevention of healthcare-associated infections and antimicrobial resistance, 1992-2008: positive trends, but perseverance needed. Infect Control Hosp Epidemiol. 2009;30(8):737-45. https://doi. org/10.1086/598682 PMID: 19566444

12. Behnke M, Gastmeier P, Geffers C, Mönch N, Reichardt C. Establishment of a national surveillance system for alcoholbased hand rub consumption and change in consumption over 4 years. Infect Control Hosp Epidemiol. 2012;33(6):618-20. https://doi.org/10.1086/665729 PMID: 22561718
13. Wetzker W, Bunte-Schönberger K, Walter J, Pilarski G, Gastmeier P, Reichardt Ch. Compliance with hand hygiene: reference data from the national hand hygiene campaign in Germany. J Hosp Infect. 2016;92(4):328-31. https://doi. org/10.1016/j.jhin.2016.01.022 PMID: 26984282

14. Hansen S, Zingg W, Ahmad R, Kyratsis Y, Behnke M, Schwab F, et al. PROHIBIT study group. Organization of infection control in European hospitals. J Hosp Infect. 2015;91(4):338-45. https://doi.org/10.1016/j.jhin.2015.07.011 PMID: 26542950

15. United Nations. Composition of macro geographic (continental) regions, geographical sub-regions, and selected economic and other groupings. New York: United Nations Statistics Division; 31 Oct 2013. [Accessed 24 Jul 2017]. Available from: http:// unstats.un.org/unsd/methods/m49/m49regin.htm

16. Organisation for Economic Co-operation and Development (OECD). Health at a glance: Europe 2012. Health expenditure in relation to GDP. Paris: OECD Publishing; 2012.

17. Pronovost P, Needham D, Berenholtz S, Sinopoli D, Chu H, Cosgrove $S$, et al. An intervention to decrease catheterrelated bloodstream infections in the ICU. N Engl J Med. 2006;355(26):2725-32. https://doi.org/10.1056/NEJMoao61115 PMID: 17192537

18. Geubbels EL, Bakker HG, Houtman P, van Noort-Klaassen MA, Pelk MS, Sassen TM, et al. Promoting quality through surveillance of surgical site infections: five prevention success stories. Am J Infect Control. 2004;32(7):424-30. https://doi. org/10.1016/j.ajic.2004.07.001 PMID: 15525920

19. Dellinger EP, Hausmann SM, Bratzler DW, Johnson RM, Daniel DM, Bunt KM, et al. Hospitals collaborate to decrease surgical site infections. Am J Surg. 2005;190(1):9-15. https://doi. org/10.1016/j.amjsurg.2004.12.001 PMID: 15972163

20. Woelber E, Schrick EJ, Gessner BD, Evans HL. Proportion of Surgical Site Infections Occurring after Hospital Discharge: A Systematic Review. Surg Infect (Larchmt). 2016;17(5):510-9. https://doi.org/10.1089/sur.2015.241 PMID: 27463235

21. European Centre for Disease Prevention and Control (ECDC). Surveillance of surgical site infections in Europe 2010-2011. Stockholm: ECDC; 2013. [Accessed 24 Jul 2017]. Available from: https://ecdc.europa.eu/en/publications-data/ surveillance-surgical-site-infections-europe-2010-2011

22. Vanhems P, Baratin D, Voirin N, Savey A, Caillat-Vallet E, Metzger $\mathrm{MH}$, et al. Reduction of urinary tract infections acquired in an intensive care unit during a 10-year surveillance program. Eur J Epidemiol. 2008;23(9):641-5. https://doi. org/10.1007/s10654-008-9270-2 PMID: 18618273

23. Zuschneid I, Schwab F, Gastmeier P, Geffers C, Behnke M, Rüden $\mathrm{H}$. Trends in ventilator-associated pneumonia rates within the German nosocomial infection surveillance system (KISS). Infect Control Hosp Epidemiol. 2007;28(3):314-8 https://doi.org/10.1086/507823 PMID: 17326022

24. Vonberg RP, Kuijper EJ, Wilcox MH, Barbut F, Tüll P, Gastmeier $P$, et al. European C difficile-Infection Control GroupEuropean Centre for Disease Prevention and Control (ECDC). Infection control measures to limit the spread of Clostridium difficile. Clin Microbiol Infect. 2008;14(Suppl 5):2-20. https://doi. org/10.1111/j.1469-0691.2008.01992.x PMID: 18412710

25. Kola A, Wiuff $C$, Akerlund $T$, van Benthem BH, Coignard $B$, Lyytikäinen 0 , et al. members of ECDIS-Net. Survey of Clostridium difficile infection surveillance systems in Europe, 2011. Euro Surveill. 2016;21(29):30291. https://doi. org/10.2807/1560-7917.ES.2016.21.29.30291 PMID: 27469420

26. van Dorp SM, Kinross P, Gastmeier P, Behnke M, Kola A, Delmée $M$, et al. European Clostridium difficile Infection Surveillance Network (ECDIS-Net) on behalf of all participants. Standardised surveillance of Clostridium difficile infection in European acute care hospitals: a pilot study, 2013. Euro Surveill. 2016;21(29):30293. https://doi.org/10.2807/15607917.ES.2016.21.29.30293 PMID: 27472820

27. Lee TB, Montgomery OG, Marx J, Olmsted RN, Scheckler WEAssociation for Professionals in Infection Control and Epidemiology. Recommended practices for surveillance: Association for Professionals in Infection Control and Epidemiology (APIC), Inc. Am J Infect Control. 2007;35(7):42740. https://doi.org/10.1016/j.ajic.2007.07.002 PMID: 17765554

28. Behnke M, Clausmeyer JO, Reichardt C, Gastmeier P. Alcohol-based hand rub consumption surveillance in German hospitals-latest results. Antimicrob Resist Infect Control. 2015;4(Suppl 1):P293. https://doi. org/10.1186/2047-2994-4-S1-P293

29. Edwards R, Sevdalis N, Vincent C, Holmes A. Communication strategies in acute health care: evaluation within the context of infection prevention and control. J Hosp Infect. 2012;82(1):259. https://doi.org/10.1016/j.jhin.2012.05.016 PMID: 22809856

30. Brannigan ET, Murray E, Holmes A. Where does infection control fit into a hospital management structure? J Hosp Infect. 
2009;73(4):392-6. https://doi.org/10.1016/j.jhin.2009.03.031

PMID: 19699008

License and copyright

This is an open-access article distributed under the terms of the Creative Commons Attribution (CC BY 4.0) Licence. You may share and adapt the material, but must give appropriate credit to the source, provide a link to the licence, and indicate if changes were made.

This article is copyright of the authors, 2018. 